\title{
First-principles studies of chromium line-ordered alloys in a molybdenum disulfide monolayer
}

\author{
N.F. Andriambelaza ${ }^{\mathrm{a}, *}$, R.E. Mapasha ${ }^{\mathrm{a}}$, N. Chettya, \\ ${ }^{a}$ Department of Physics, University of Pretoria, Pretoria 0002, South Africa \\ ${ }^{b}$ National Institute for Theoretical Physics, Johannesburg, 2000, South Africa
}

\begin{abstract}
Density functional theory calculations have been performed to study the thermodynamic stability, structural and electronic properties of various chromium $(\mathrm{Cr})$ line-ordered alloy configurations in a molybdenum disulfide $\left(\mathrm{MoS}_{2}\right)$ hexagonal monolayer for band gap engineering. Only the molybdenum (Mo) sites were substituted at each concentration in this study. For comparison purposes, different $\mathrm{Cr}$ line-ordered alloy and random alloy configurations were studied and the most thermodynamically stable ones at each concentration were identified. The configurations formed by the nearest neighbor pair of $\mathrm{Cr}$ atoms are energetically most favorable. The line-ordered alloys are constantly lower in formation energy than the random alloys at each concentration. An increase in $\mathrm{Cr}$ concentration reduces the lattice constant of the $\mathrm{MoS}_{2}$ system following the Vegard's law. From density of states analysis, we found that the $\mathrm{MoS}_{2}$ band gap is tunable by both the $\mathrm{Cr}$ line-ordered alloys and random alloys with the same magnitudes. The reduction of the band gap is mainly due to the hybridization of the $\mathrm{Cr} 3 d$ and Mo $4 d$ orbitals at the vicinity of the band edges. The band gap engineering and magnitudes $(1.65 \mathrm{eV}$ to $0.86 \mathrm{eV})$ suggest that the $\mathrm{Cr}$ alloys in a $\mathrm{MoS}_{2}$ monolayer are good candidates for nanotechnology devices.
\end{abstract}

Keywords: two dimensional material, molybdenum disulfide, density functional theory, alloys.

\section{Introduction}

In the last few years, two dimensional (2D) layered materials have become an important area of research in material science. Due to their reduced dimensionality, they possess unique electronic and optical properties different from their bulk counterparts [1, 2, 3, 4]. These fascinating properties make 2D materials suitable candidates for various applications in nanoelectronic technologies $[2,4,5]$. The successful synthesis of graphene [6] was the starting point for the extensive exploration of different types of 2D materials. Graphene has extremely high charge carrier mobility and exceptional mechanical flexibility [1, 4]. However, it is a zero band gap material [1]. Consequently, this limits the direct application of

\footnotetext{
${ }^{*}$ Corresponding author

Email address: arinala.f@gmail . com (N.F. Andriambelaza)
}

graphene in the nanotechnological devices. Many experimental and theoretical efforts have been made to open a band gap in a graphene system $[7,8,9,10,11]$. A sizable band gap was achieved, but it was found to be either too small $[7,8,9]$ or too large $[10,11]$. This issue drove researchers to explore other 2D materials such as boronitrene (h-BN) [12] and transition metal dichalcogenides (TMD) [13]. Some of the TMD 2D systems are particularly interesting due to their tremendous properties such as high mechanical strength, direct band gap and optical transparency $[14,15,16,17]$. Because of these exotic properties, TMD materials are suitable for applications in the nanoelectronic and optoelectronic devices [15, 18, 19].

TMD materials have the chemical formula $\mathrm{MX}_{2}$, where $\mathrm{M}$ is a transition metal element and $\mathrm{X}$ stands for chalcogen from group VIB element. Depending on the type of the transition element and the chalcogen, TMD 2D systems can be an insu- 
lator (e.g. Zirconium disulfide $\left.\left(\mathrm{ZrS}_{2}\right)\right)$, metal (e.g. Niobium disulfide $\left(\mathrm{NbS}_{2}\right)$ ), semimetal (e.g. Tungsten telluride $\left(\mathrm{WTe}_{2}\right)$ ) and semiconductor (e.g. Chromium disulfide $\left(\mathrm{CrS}_{2}\right)$, Molybdenum disulfide $\left(\mathrm{MoS}_{2}\right)$ and Tungsten disulfide $\left.\left(\mathrm{WS}_{2}\right)\right)$ [20]. Amongst the various TMD monolayers, $\mathrm{MoS}_{2}$ monolayer is the most widely studied material owing to some of its special semiconducting properties similar to those of carbon-based graphene [21, 22]. As in other semiconductors, the electronic and optical properties of $\mathrm{MoS}_{2} 2 \mathrm{D}$ material are usually controllable by tuning the band gap. Thus, engineering the band gap of $\mathrm{MoS}_{2}$ monolayer is important for designing nanoelectronic devices.

Historically, alloying has been used as an effective method to tune electronic structures of 3D semiconductor materials [23, 24, 25]. Recently, several experimental and theoretical studies have investigated the formation possibilities and electronic properties of alloys in the 2D TMD materials [26, 27, 28, 29]. For instance, Xu et al. [26] reported a chemical vapor deposition (CVD) strategy for the growth of $\mathrm{Mo}_{1-x} \mathrm{~W}_{x} \mathrm{~S}_{2}$ and $\mathrm{MoS}_{1-x} \mathrm{Se}_{x}$ monolayers. It was found that $\mathrm{W}(\mathrm{Se})$ alloys increase (reduce) the band gap of pristine $\mathrm{MoS}_{2}$ monolayer from $1.83 \mathrm{eV}$ to $1.97 \mathrm{eV}(1.55 \mathrm{eV})$. It was suggested that this band gap engineering is important to further optimize the performance of nanoscale photoelectronic devices. Tongay et al. [29] successfully synthesized $\mathrm{Mo}_{1-x} \mathrm{~W}_{x} \mathrm{Se}_{2}$ and reported that the band gap of a $\mathrm{MoS}_{2}$ monolayer can be tuned by varying the $\mathrm{W}$ concentration. Using density functional theory (DFT) approach, Xi et al. [27] reported that the band gap of $\mathrm{MoS}_{2}$ monolayer is tunable by $\mathrm{W}$ alloying and its enthalpy of formation is exothermic. Komsa et al. [28] investigated the thermodynamic stability and electronic properties of random $\mathrm{Mo}_{1-x} \mathrm{~W}_{x} \mathrm{~S}_{2}, \mathrm{MoS}_{1-x} \mathrm{Se}_{x}, \mathrm{MoS}_{1-x} \mathrm{Te}_{x}$ and $\mathrm{MoSe}_{1-x} \mathrm{Te}_{x}$ alloys using DFT. They found that the formation energies for $\mathrm{MoS}_{1-x} \mathrm{Te}_{x}$ and $\mathrm{MoSe}_{1-x} \mathrm{Te}_{x}$ alloys are positive although very small and for $\mathrm{Mo}_{1-x} \mathrm{~W}_{x} \mathrm{~S}_{2}$ and $\mathrm{MoS}_{1-x} \mathrm{Se}_{x}$ are negative. They also noted that the chalcogen alloys usually reduce the band gap of a $\mathrm{MoS}_{2}$ monolayer, whereas $\mathrm{W}$ alloys raise it during the increase in concentration. In general, Se, Te and $\mathrm{W}$ alloys in a $\mathrm{MoS}_{2}$ monolayer are exothermic and fine tune the band gap. This band gap engineering in a $\mathrm{MoS}_{2}$ system is essential for the fabrication of nanoelectronic devices. Chromium $(\mathrm{Cr})$ is in the same periodic table group with Mo and $\mathrm{W}$, and thus deserve to be investigated as well. Xie [30] reported that creating TMD alloys within the same transition metal group is usually feasible. This has been noted in the case of W replacing Mo or Se and Te replacing $\mathrm{S}$ in a $\mathrm{MoS}_{2}$ monolayer. These type of systems have a very small lattice mismatch with the $\mathrm{MoS}_{2}$ monolayer. To the best of our knowledge, there is no detailed publications on the study of $\mathrm{Mo}_{1-x} \mathrm{Cr}_{x} \mathrm{~S}_{2}$ and the transition from $\mathrm{MoS}_{2}$ to $\mathrm{CrS}_{2}$ monolayer through $\mathrm{Cr}$ alloying.

Studying all the possible configurations of alloys in a $\mathrm{MoS}_{2}$ monolayer using a DFT method is not a tractable task. Alloys in a $2 \mathrm{D}$ materials can appear in different configurations such as clusters (many shapes : triangle-like, square, circular, etc), lines and can randomly scattered away from each other in different forms. The number of possible distinct configurations at any concentration with a number $n$ of the $\mathrm{Cr}$ atoms is obtained by :

$$
\frac{N !}{n !(N-n) !},
$$

where $\mathrm{N}$ is the total number of Mo sites in a monolayer. For instance, in the case of $20 \%$ concentration constituted by a total of $5 \mathrm{Cr}$ atoms replacing the Mo atoms in a $5 \times 5$ supercell, there are 53130 possible configurations. Computing all of these possible configurations is practically impossible as this requires huge computational resources. To reduce this difficulty, adopting typical configurations is helpful. Experimentally, it was reported that the TMD alloys are usually synthesized in random phases at high temperatures [30]. However, DFT studies at 0 Kelvin have reported that ordered phases have a lower formation energy than random phases [30, 31, 32]. Most of the previous studies on TMD alloys considered various cluster configurations using a DFT approach [30, 31, 33]. A line alloy which is another form of ordered alloy can appear in different configurations in a $\mathrm{MoS}_{2}$ monolayer, therefore worth to be studied and to know the most energetically favorable configuration. Komsa et al. [34] have studied line-ordered alloys of the 
Se atoms substituting the $\mathrm{S}$ atoms in a $\mathrm{MoS}_{2}$ monolayer. They stated that line-ordered alloy can be a way to alter the electronic properties of $\mathrm{MoS}_{2}$ monolayer. The knowledge of the transition metal line-ordered alloys substituting the Mo sites is scarce.

In this paper, we present a comparative study of the physical properties of line-ordered alloys and random alloys of $\mathrm{Cr}$ atoms in a $\mathrm{MoS}_{2}$ monolayer using a DFT approach. In a $\mathrm{MoS}_{2}$ monolayer supercell, the possible line-ordered alloy configurations are few and can easily be identified at each $\mathrm{Cr}$ concentration. However, for the random alloys, a large number of different configurations are possible. Special quasi-random structure (SQS) method [35] is a tool that can mimic the possible random alloy configurations at each concentration. This method has been successfully applied to various alloys in a $\mathrm{MoS}_{2}$ monolayer [33].

Based on the alloy formation energy analysis, we identify the lowest energy configurations for line-ordered alloys as well as random alloys at each concentration, when the $\mathrm{Cr}$ atoms substitute the Mo atoms. To have an insight to the electronic properties of the lowest energy configurations, the densities of states (DOS) were plotted. As opposed to $\mathrm{W}$ alloys, the band gap of $\mathrm{Mo}_{1-x} \mathrm{Cr}_{x} \mathrm{~S}_{2}$ decreases with the increase in $\mathrm{Cr}$ concentration. The band gap of $\mathrm{MoS}_{2}$ monolayer is finite but tunable, making it a good candidate in nanoelectronic devices.

\section{Methodology}

We have systematically investigated the thermodynamic stability, the structural and electronic properties of $\mathrm{Mo}_{1-x} \mathrm{Cr}_{x} \mathrm{~S}_{2}$ alloys using the DFT method implemented in the Vienna $a b$ initio simulation package (VASP) [36, 37]. To describe the core electron interactions, projector augmented wave (PAW) pseudopotential [38] were used. The generalized gradient approximation (GGA) exchange correlation parameterized by Perdew, Burke, and Ernzerhof (PBE) [39] was employed to treat the exchange correlation interactions. The supercell size, kinetic energy cut-off and Brillouin zone sampling convergence tests were conducted, and a $5 \times 5$ supercell was chosen. A kinetic energy cutoff of $300 \mathrm{eV}$ for the plane wave expansion and
$2 \times 2 \times 1 \mathrm{k}$-point mesh were used. All the structures were fully relaxed. The convergence threshold was $10^{-5} \mathrm{eV}$ for energies and $2 \times 10^{-2} \mathrm{eV} / \AA$ for forces. To suppress interactions between adjacent supercells, a vacuum spacing of $15 \AA$ was constructed in the perpendicular direction.

To examine the relative stability of the distinct possible configurations of $\mathrm{Mo}_{1-x} \mathrm{Cr}_{x} \mathrm{~S}_{2}$ alloys, the formation energy of each configuration was evaluated. The formation energy $\left(\mathrm{E}_{\text {form }}\right)$ is given by:

$$
E_{\text {form }}=E_{M o_{1-x} C r_{x} S_{2}}-(1-x) E_{M o S_{2}}-x E_{C r S_{2}}
$$

where $\mathrm{E}_{M o_{1-x} C r_{x} S_{2}}, \mathrm{E}_{M o S_{2}}$ and $\mathrm{E}_{C r S_{2}}$ are the total energies of the mixed compound, the pristine $\mathrm{MoS}_{2}$ and the pristine $\mathrm{CrS}_{2}$ monolayers, respectively. The $x$ parameter is the concentration of $\mathrm{Cr}$ introduced in a $\mathrm{MoS}_{2}$ monolayer [40].

The different flavors of GGA functional [39, 41, 42, 43, 44] are known to underestimate the energy band gap of a material. In order to improve our band gap values, the hybrid functional of Heyd, Scuseria, and Ernzerhof (HSE06) [45] was also used. At the end of our discussion, we briefly compare the band gap values obtained from GGA functional with those obtained from the HSE functional.

The $\mathrm{Cr}$ substitution alloys in a $\mathrm{MoS}_{2}$ monolayer $\left(\mathrm{Mo}_{1-x} \mathrm{Cr}_{x} \mathrm{~S}_{2}\right)$ can be generalized as a binary $\mathrm{A}_{1-x} \mathrm{~B}_{x}$ alloy. For a perfect random alloy, the correlation function is given by $\prod_{k, m}(\mathrm{R})=(2 x-1)^{k}$, where $k=2,3, \ldots$ indicates the pair (2), triple (3),... correlation functions; $m=1,2,3, \ldots$ indicates the first, second and third,...,nearest-neighbor distances and $x$ represent the concentration of the substituted atoms [46]. In the SQS method, the sites $i$ occupied by atoms A or B are assigned to a variable $\sigma_{i}$ equals to -1 or 1 , respectively. The correlation function for this binary alloy can be written as $\prod_{k, m}=\frac{1}{N_{k, m}} \sum_{\{k, m\}} \sigma_{1} \sigma_{2} \ldots \sigma_{k}$, where $N_{k, m}$ is the total number of shape that can be obtained when varying $k$ and $m$. In the present study, only the nearest-neighbor pairs are scrutinized, since the interactions between far distant neighbors have been reported not to have much effect on the total energy [33]. In this model (nearest neighbor pair), the correlation function 
$\Pi_{2,1}$ is given by $\Pi_{2,1}=\frac{1}{N_{\text {bond }}} \sum_{j, h=1, N}^{j>h} \sigma_{j} \sigma_{h}$, where $N_{\text {bond }}$ is the number of bonds between nearest neighbor metals, $N$ is the total number of transition metal in the supercell, $j$ and $h$ represent the two pair sites considered. If the total number of the nearest-neighbor bonds between $A-A$ (Mo-Mo), $B-B$ (Cr-Cr) and $A-B$ (Mo-Cr) atoms are denoted by $N_{A A}, N_{B B}$ and $N_{A B}$, respectively, the correlation function can be written as :

$$
\Pi_{2,1}=\frac{N_{A A}+N_{B B}-N_{A B}}{N_{A A}+N_{B B}+N_{A B}} .
$$

The number of atoms $A(\mathrm{Mo})$ and $B(\mathrm{Cr})$ in an $\mathrm{A}_{1-x} \mathrm{~B}_{x}$ alloy is given by: $N_{A}=N(1-x)$ and $N_{B}=N x$. In addition, assuming that $z$ is the coordination number of atoms in the system, $N_{A}$ and $N_{B}$ are related to the number of bonds as:

$$
\left\{\begin{array}{l}
N_{A}=\frac{N_{A B}+2 N_{A A}}{z} \\
N_{B}=\frac{N_{A B}+2 N_{B B}}{z}
\end{array}\right.
$$

Therefore, the correlation function in equation. 3 becomes :

$$
\Pi_{2,1}=1-4 x+\frac{8 N_{B B}}{N z} .
$$

Thus, equation. 5 shows that the correlation function can be completely described by $N_{B B}$. The SQS configurations are considered to have the same correlation functions as the perfect random alloys: $\prod_{2,1}(R)=\prod_{2,1}(S Q S)$ [46]. Using this equality and equation. 5 , we found that the number of $N_{B B}=\frac{1}{2} x^{2} N z$ in a TMD monolayer. In our study, $N=25$ and $z=6$ in a $5 \times 5$ supercell of $\mathrm{MoS}_{2}$ monolayer. It can be shown that at $x=0.2,0.4,0.6$ and 0.8 the $N_{B B}=3,12,27$ and 48 respectively, yielding the SQS random alloy configurations. Many random alloys configurations are possible but the optimum SQS configurations are those that have the same correlation function as the perfect random alloy, i.e, pair, triple, etc.

\section{Results and discussion}

In order to study the effect of the Cr substitutional dopants on the thermodynamic stability, structural and electronic properties of a $\mathrm{MoS}_{2}$ monolayer, the physical properties of the pristine $\mathrm{MoS}_{2}$ and $\mathrm{CrS}_{2}$ monolayers need to be understood first. In this section, we investigate the structural and electronic properties of these pristine systems. Thereafter, the $\mathrm{Cr}$ atoms are introduced into a $\mathrm{MoS}_{2}$ monolayer by occupying the Mo sites only. An incorporation of the $\mathrm{Cr}$ atoms into a $\mathrm{MoS}_{2}$ monolayer is treated in two cases; firstly, the low $\mathrm{Cr}$ concentration has been considered and secondly, the high concentration has been taken into account to form the alloys.

\subsection{Pristine $\mathrm{MoS}_{2}$ and $\mathrm{CrS} \mathrm{S}_{2}$ monolayers}

(a)

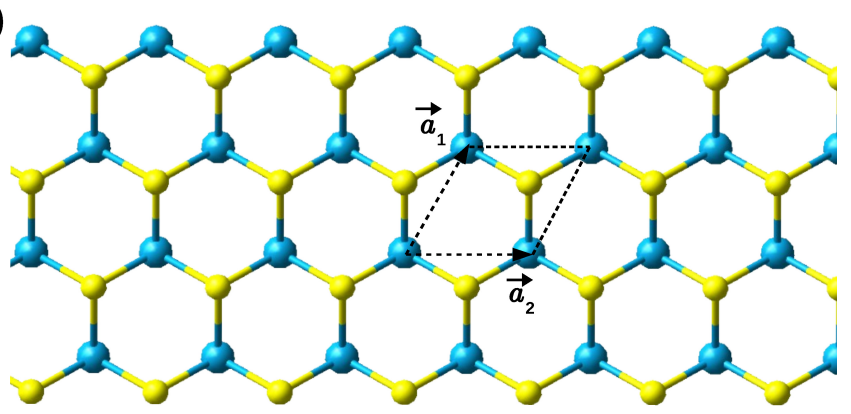

(b)

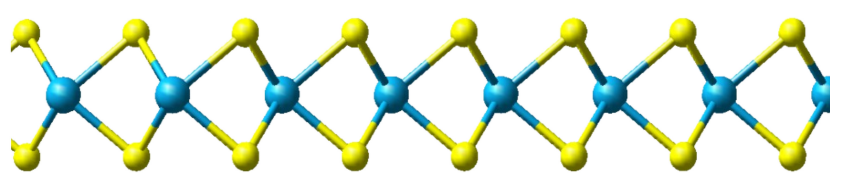

Figure 1: (a) Top view and (b) side view of the 2D TMD MoS 2 or $\mathrm{CrS}_{2}$. The blue spheres indicate the transition metal atoms and the light yellow spheres indicate the sulfur (S) atoms. $\overrightarrow{a_{1}}$ and $\overrightarrow{a_{2}}$ are the lattice vectors. A unit cell is drawn with dashed lines.

The 2D TMD $\left(\mathrm{MoS}_{2}\right.$ and $\left.\mathrm{CrS}_{2}\right)$ have different polymorphs such as the hexagonal and tetragonal [47]. Previous studies reported that the hexagonal structures of $\mathrm{MoS}_{2}$ and $\mathrm{CrS}_{2}$ monolayers are the most stable ones [20]. Top view and side view of the hexagonal TMD monolayer are shown in figure. 1. The transition metal (Mo or $\mathrm{Cr}$ ) layer is sandwiched between the two adjacent sulfur (S) layers and form covalent bonds. The transition metal atoms occupy one sublattice of the hexagon and the $\mathrm{S}$ atoms occupy the other.

The fully optimized lattice constant for the $\mathrm{MoS}_{2}$ and $\mathrm{CrS}_{2}$ monolayers are found to be $3.18 \AA$ and $3.05 \AA$, respectively. The bond lengths Mo-S and Cr-S are $2.41 \AA$ and $2.29 \AA$, re- 

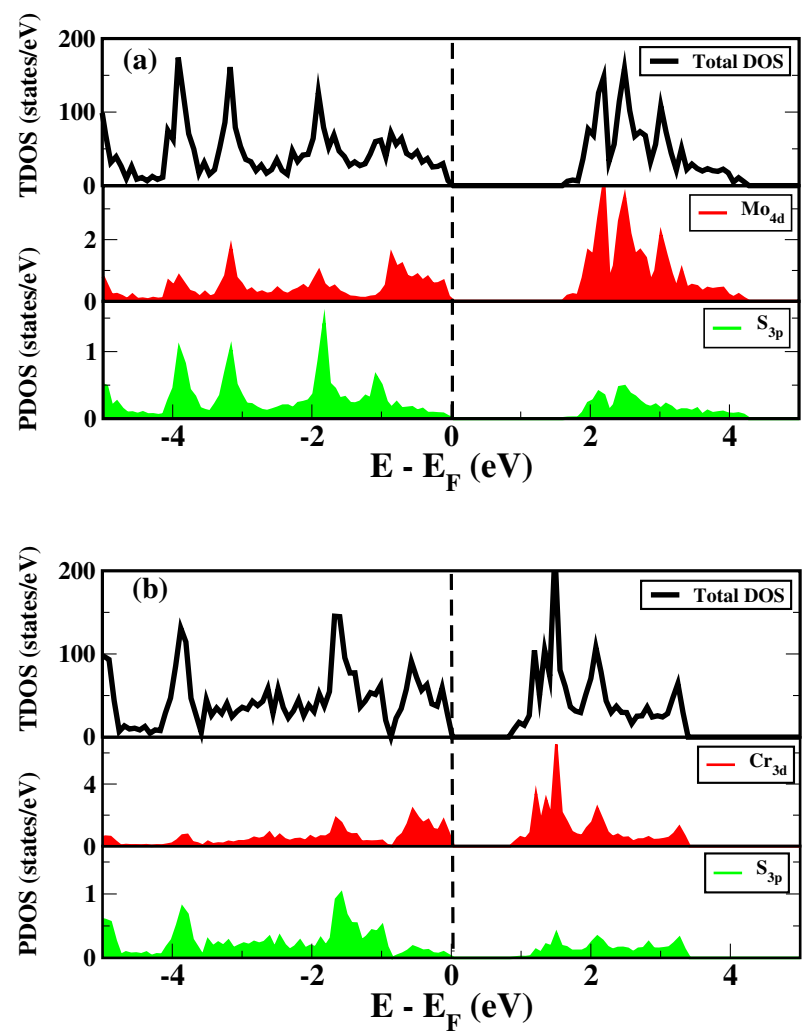

Figure 2: The total density of states (TDOS) for the pristine (a) $\mathrm{MoS}_{2}$ and (b) $\mathrm{CrS}_{2}$ monolayers (black lines). The red and green curves are the projected density of states (PDOS) for the transition metal $d$ orbitals and S $3 p$ orbitals, respectively. The dashed vertical lines indicate the Fermi level.

spectively. These values agree quite well with the experimental and the previous DFT data $[48,49,50]$.

To study the electronic properties of the pristine $\mathrm{MoS}_{2}$ and $\mathrm{CrS}_{2}$ monolayers, density of states (DOS) were evaluated. Figure. 2 shows the total DOS (TDOS) for pristine $\mathrm{MoS}_{2}$ (figure. 2(a)) and $\mathrm{CrS}_{2}$ (figure. 2(b)) monolayers. These plots present a gap between the top of the valence band (VBM) and the bottom of the conduction band (CBM) indicating that both the pristine systems are semiconductors. We found that the band gaps of $\mathrm{MoS}_{2}$ and $\mathrm{CrS}_{2}$ monolayers are $1.65 \mathrm{eV}$ and $0.86 \mathrm{eV}$, respectively. These values are consistent with the previous theoretical and experimental data shown in Table. 1 . The PDOS in figure. 2 show that the VBM and CBM states of $\mathrm{MoS}_{2}$ and $\mathrm{CrS}_{2}$ monolayers arise mainly from the $4 d$ and $3 d$ orbitals of the transition metal, respectively. Small contribution from the $3 p$ orbitals of the $\mathrm{S}$ atoms is noted in both figures. This is in agreement with the results of Dolui et al. [55] and Zhuang
Table 1: The theoretical and experimental energy band gaps of the pristine $\mathrm{MoS}_{2}$ and $\mathrm{CrS}_{2}$ monolayers.

\begin{tabular}{llll}
\hline Structure & Method & Band gap (eV) & Ref. \\
\hline $\mathrm{MoS}_{2}$ & Experimental & 1.9 & {$[51]$} \\
& Experimental & 1.75 & {$[52]$} \\
& GGA & 1.67 & {$[49]$} \\
& GGA & 1.65 & This work \\
& HSE & 2.25 & {$[49]$} \\
& HSE & 2.14 & {$[53]$} \\
$\mathrm{CrS}_{2}$ & HSE & 2.17 & This work \\
& GGA & 0.89 & {$[33]$} \\
& GGA & 0.86 & This work \\
& HSE & 1.48 & {$[54]$} \\
& HSE & 1.31 & This work \\
\hline
\end{tabular}

et al. [54].

Spin polarized calculations were carried out for all of the calculations in this paper. However, the majority spin DOS are symmetrical to the minority spin DOS, indicating that the systems are non magnetic in nature $[33,56]$. Therefore, we only show the DOS for spin-up channel, for the entire paper.

\subsection{Isolated $\mathrm{Cr}$ dopants in a $\mathrm{MoS}_{2}$ monolayer}

Next, we examine the effect of isolated $(I)$ one and two $\mathrm{Cr}$ dopants on the thermodynamic stability, structural and electronic properties of a $\mathrm{MoS}_{2}$ monolayer. As mentioned earlier, the Mo and S atoms occupy different sublattices of the hexagonal $\mathrm{MoS}_{2}$ monolayer as shown in figure. 1 .

The Mo atoms occupy one sublattice for the entire structure, and due to the symmetry of the system, we only have one possible unique configuration for single $\mathrm{Cr}$ doping shown in figure. 3(a) called $\mathrm{C}_{1(I)}$. However, in the case of the two $\mathrm{Cr}$ dopants, different configurations are possible.

All the distinct configurations of the two $\mathrm{Cr}$ dopants are shown in figure. 3(b)-(e), namely configurations $\mathrm{C}_{2(I)}, \mathrm{C}_{3(I)}$, $\mathrm{C}_{4(I)}$ and $\mathrm{C}_{5(I)}$. Configuration $\mathrm{C}_{2(I)}$ is constructed by introducing the two $\mathrm{Cr}$ atoms at the Mo sites within the same hexagonal ring-like in a $5 \times 5$ supercell of a $\mathrm{MoS}_{2}$ monolayer. These 


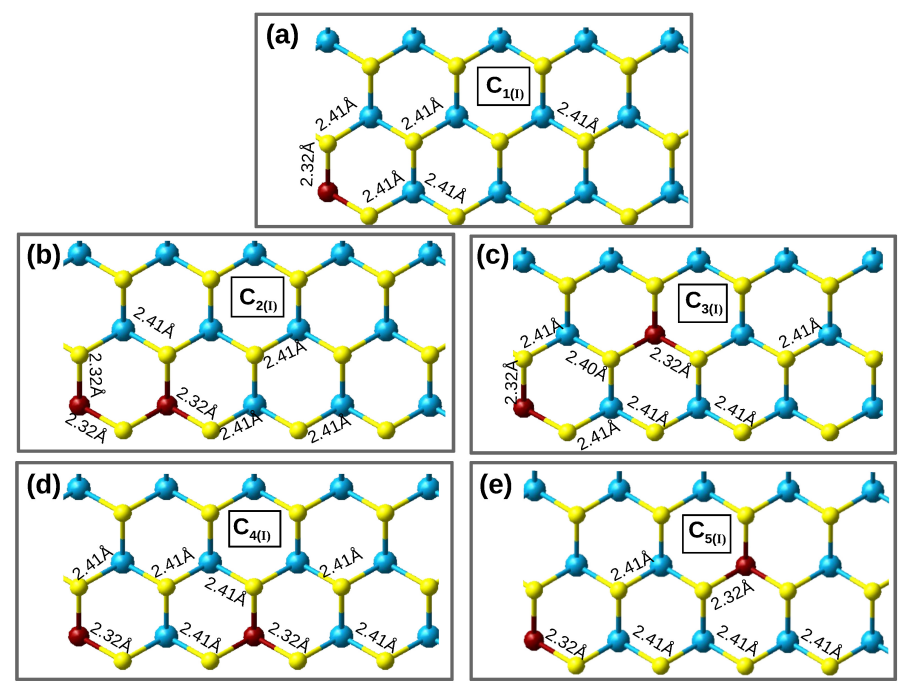

Figure 3: (a) The relaxed structure of a single $\mathrm{Cr}$ doping in a $\mathrm{MoS}_{2}$ monolayer. The various configurations of the two $\mathrm{Cr}$ dopants (b) $\mathrm{C}_{2(I)}$, (c) $\mathrm{C}_{3(I)}$, (d) $\mathrm{C}_{4(I)}$ and (e) $\mathrm{C}_{5(I)}$. The blue, red and light yellow spheres indicate the $\mathrm{Mo}, \mathrm{Cr}$ and $\mathrm{S}$ atoms, respectively.

two dopants are first nearest neighbor to each other (see figure. 3(b)). The second possible configuration $\mathrm{C}_{3(I)}$ involves the second hexagonal ring-like. $\mathrm{C}_{3(I)}$ is obtained when the second $\mathrm{Cr}$ atom is the second nearest neighbor of the first $\mathrm{Cr}$ atom (see figure. 3(c)). Configuration $\mathrm{C}_{4(I)}$ is obtained when the second $\mathrm{Cr}$ atom is placed as the third nearest neighbor with the first $\mathrm{Cr}$ atom (see figure. $3(\mathrm{~d})$ ). The last configuration $\mathrm{C}_{5(I)}$ is obtained when the second atom is the forth nearest neighbor of the first $\mathrm{Cr}$ atom (see figure. 3(e)) considering only the Mo sites.

In order to study the stability of these different configura-

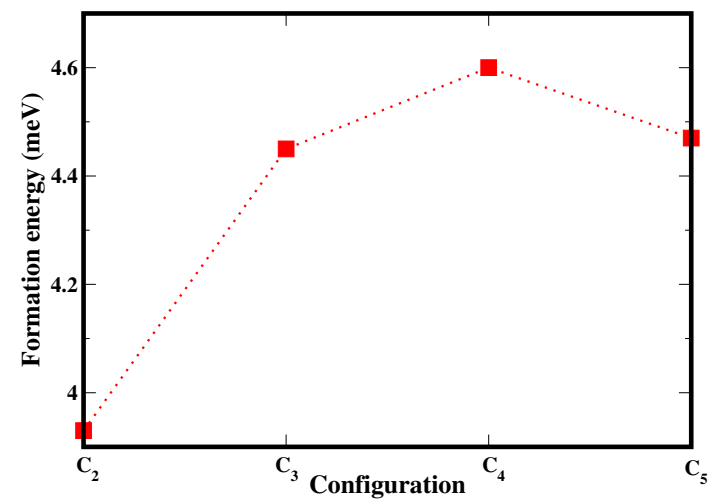

Figure 4: The formation energies for distinct configurations of the two dopants in a $\mathrm{MoS}_{2}$ monolayer. Along the $\mathrm{x}$ axis, configurations $\mathrm{C}_{2(I)}, \mathrm{C}_{3(I)}, \mathrm{C}_{4(I)}$ and $\mathrm{C}_{5(I)}$ denote the first, second, third and forth nearest neighbor $\mathrm{Cr}$ atoms. tions, we calculated the formation energies using equation. 2 . The formation energy for $\mathrm{C}_{1(I)}$ is $2 \mathrm{meV}$, revealing an endothermic reaction. Figure. 4 summarizes the formation energies for the different configurations of the two dopants in a $\mathrm{MoS}_{2}$ monolayer $\left(\mathrm{C}_{2(I)}, \mathrm{C}_{3(I)}, \mathrm{C}_{4(I)}\right.$ and $\left.\mathrm{C}_{5(I)}\right)$. It can be seen that $\mathrm{C}_{4(I)}$ has the highest formation energy, while $\mathrm{C}_{2(I)}$ possessing the lowest. We realize that the $\mathrm{Cr}$-doped system becomes more unstable when the $\mathrm{Cr}$ atoms move farther away from each other. Thus, this is an evidence that the two $\mathrm{Cr}$ dopants prefer to be closer to each other in a $\mathrm{MoS}_{2}$ supercell, when considering the Mo sites only. Although positive, the formation energies shown in figure. 4 are very low, suggesting that $\mathrm{Cr}$-doped configurations can form under reasonable conditions.

In all configurations considered, the $\mathrm{Cr}-\mathrm{S}$ bond distance is $0.10 \AA$ on average lower than the initial bond length of the $\mathrm{MoS}_{2}$ monolayer. Various bond lengths can be read from figure. 3. This relatively small value reveals that $\mathrm{Cr}$ does not cause significant structural distortions in a $\mathrm{MoS}_{2}$ monolayer.

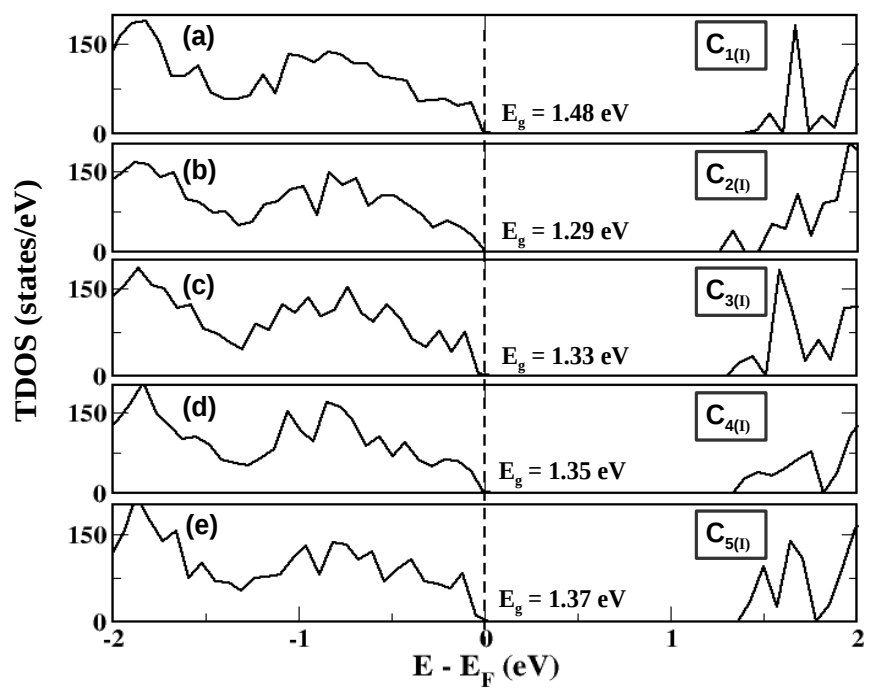

Figure 5: (a) The TDOS of single $\mathrm{Cr}$ doping in a $\mathrm{MoS}_{2}$ monolayer $\left(\mathrm{C}_{1(I)}\right)$. The TDOS of the configurations (b) $\mathrm{C}_{2(I)}$, (c) $\mathrm{C}_{3(I)}$, (d) $\mathrm{C}_{4(I)}$ and (e) $\mathrm{C}_{5(I)}$. The black dashed vertical lines indicate the Fermi level.

Figure. 5 presents the TDOS of our distinct $\mathrm{Cr}$ configurations in a $\mathrm{MoS}_{2}$ monolayer. It is found that the incorporation of the $\mathrm{Cr}$ dopants preserves the semiconducting properties of 

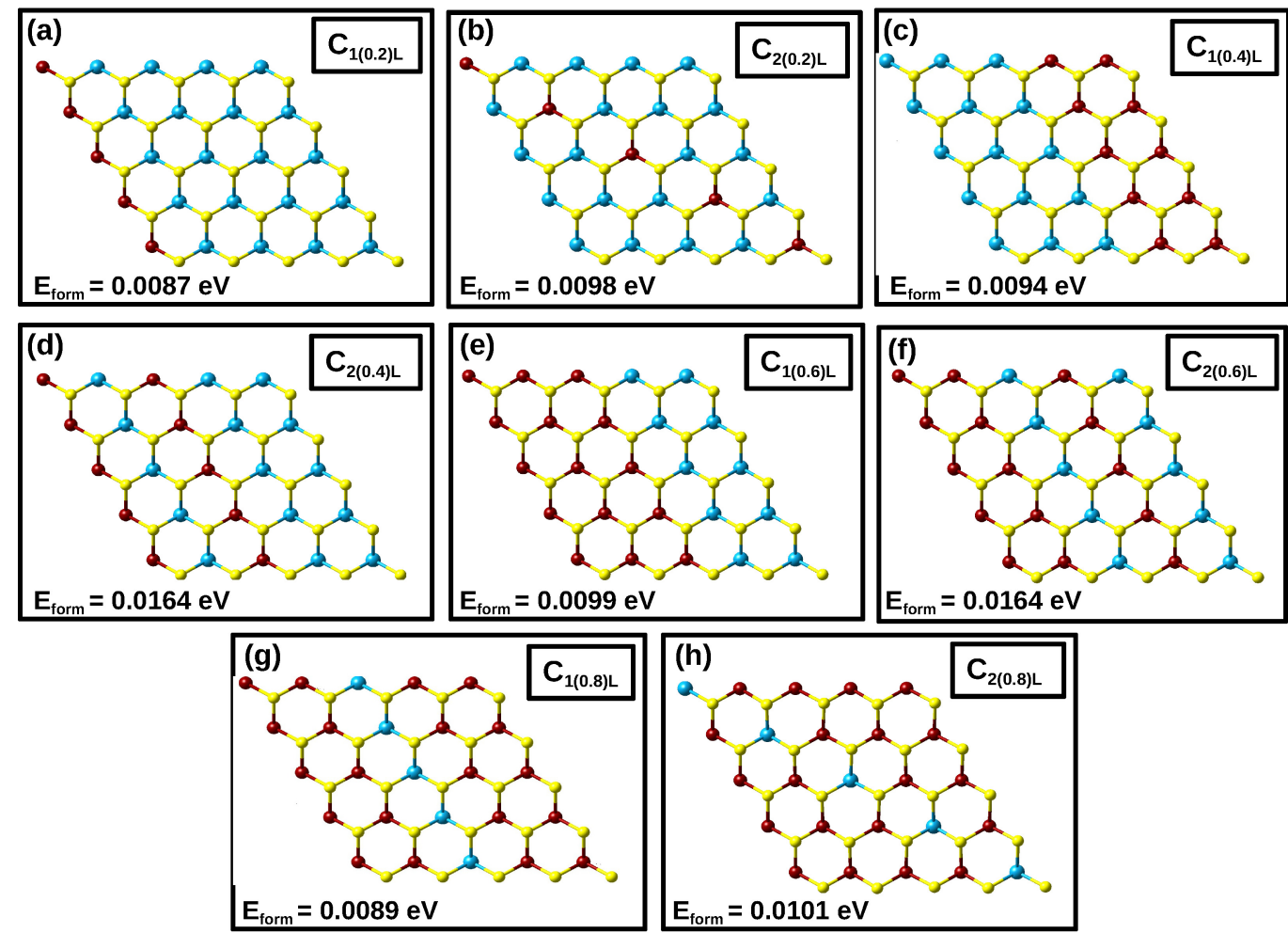

Figure 6: All the possible configurations of line-ordered alloys of $\mathrm{Mo}_{1-x} \mathrm{Cr}_{x} \mathrm{~S}_{2}$ at each concentration. Configurations (a) $\mathrm{C}_{1(0.2) L}$ and (b) $\mathrm{C}_{2(0.2) L}$ correspond to 20\%, (c) $\mathrm{C}_{1(0.4) L}$ and (d) $\mathrm{C}_{2(0.4) L}$ to $40 \%$, (e) $\mathrm{C}_{1(0.6) L}$ and (f) $\mathrm{C}_{2(0.6) L}$ to $60 \%$, and (g) $\mathrm{C}_{1(0.8) L}$ and (h) $\mathrm{C}_{2(0.8) L}$ to $80 \%$ of the Cratoms. The blue, red and yellow spheres represent the $\mathrm{Mo}, \mathrm{Cr}$ and $\mathrm{S}$ atoms, respectively. The formation energy $\left(\mathrm{E}_{f o r m}\right)$ of each configuration obtained using equation. 2 is shown at the bottom of each diagram.

$\mathrm{MoS}_{2}$ monolayer but reduces the band gap of the system significantly. Even at a very low concentration of $4 \% \mathrm{Cr}$ dopant (one $\mathrm{Cr}$ dopant), the band gap has been reduced from $1.65 \mathrm{eV}$ (pristine $\mathrm{MoS}_{2}$ ) to $1.48 \mathrm{eV}$. Comparing different configurations of the two dopants, it is noted that the most stable configuration $\mathrm{C}_{2(I)}$ has the smallest band gap (see figure. $5 \mathrm{~b}$ ), while $\mathrm{C}_{5(I)}$ possessing the largest (see figure. 5e). Revealing that the band gap can be fine tuned by variation of the distance between the two Cr dopants.

Since these preliminary results suggest that the $\mathrm{Cr}$ atoms prefer to be closer to each other, it is important to examine the effect of $\mathrm{Cr}$ dopants at high concentrations. In this study, the $\mathrm{Cr}$ line-ordered alloy and random alloy at different concentrations and in various configurations are studied. This is further supported by Lewis et al. [57] who reported that Cr substituting Mo in a $\mathrm{MoS}_{2}$ monolayer can be synthesized at high concentration.

\subsection{Line-ordered and random alloys of $\mathrm{Cr}$ atoms in a $\mathrm{MoS}_{2}$} monolayer

\subsubsection{Thermodynamic stability and structural properties of $M o_{1-x} C r_{x} S_{2}$ alloys}

As mentioned earlier, simulation of the alloys in a layered systems is a great challenge due to thousands of possible atomic arrangements. In this section, a systematic study of $\mathrm{Cr}$ lineordered alloys and random alloys has been considered. Five concentrations $(x=0.2, x=0.4, x=0.6$ and $x=0.8$ correspond to $20 \%, 40 \%, 60 \%, 80 \%$ and $100 \% \mathrm{Cr}$ atoms) have been chosen.

To create the line-ordered alloys, we substitute the Mo atoms in a $\mathrm{MoS}_{2}$ monolayer with the $\mathrm{Cr}$ atoms at different concentrations, along a particular direction (zigzag and diagonal). At each concentration, distinct configurations are identified and examined. All the possible $\mathrm{Cr}$ configurations are shown in fig- 

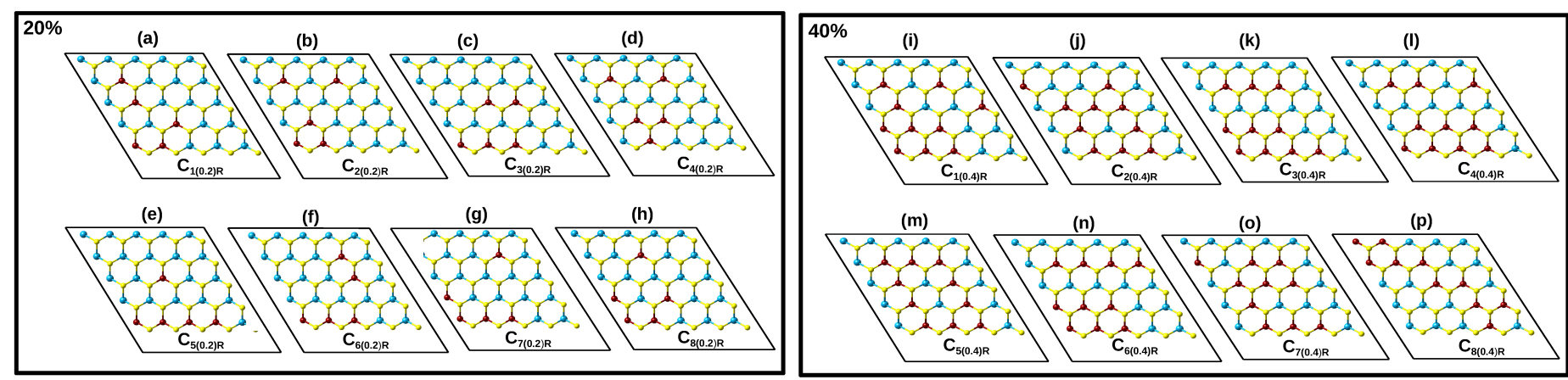

Figure 7: Various selected unique $\mathrm{Cr}$ random alloy configurations at $20 \%$ of $\mathrm{Cr}$ atoms (left panel): (a) $\mathrm{C}_{1(0.2) R}$, (b) $\mathrm{C}_{2(0.2) R}$, (c) $\mathrm{C}_{3(0.2) R}$, (d) $\mathrm{C}_{4(0.2) R}$, (e) $\mathrm{C}_{5(0.2) R}$, (f) $\mathrm{C}_{6(0.2) R}$, (g) $\mathrm{C}_{7(0.2) R}$ and (h) $\mathrm{C}_{8(0.2) R}$. Those obtained at $40 \%$ (right panel) are : (i) $\mathrm{C}_{1(0.4) R}$, (j) $\mathrm{C}_{2(0.4) R},(\mathrm{k}) \mathrm{C}_{3(0.4) R}$, (l) $\mathrm{C}_{4(0.4) R}$, (m) $\mathrm{C}_{5(0.4) R}$, (n) $\mathrm{C}_{6(0.4) R}$, (o) $\mathrm{C}_{7(0.4) R}$ and (p) $\mathrm{C}_{8(0.4) R}$. At $60 \%$ and $80 \%$ concentrations, the selected configurations are the same as those obtained at $40 \%$ and $20 \%$, respectively, but with transition metals swapped. The blue, red and yellow spheres represent the $\mathrm{Mo}, \mathrm{Cr}$ and $\mathrm{S}$ atoms, respectively.

ure. 6 named $\mathrm{C}_{1(0.2) L}, \mathrm{C}_{2(0.2) L}, \mathrm{C}_{1(0.4) L}, \mathrm{C}_{2(0.4) L}, \mathrm{C}_{1(0.6) L}, \mathrm{C}_{2(0.6) L}$, $\mathrm{C}_{1(0.8) L}$ and $\mathrm{C}_{1(0.8) L}$. To avoid repetition, the periodic boundary conditions are always taken into account when constructing the line-ordered alloys. Configurations $\mathrm{C}_{1(0.2) L}$ and $\mathrm{C}_{2(0.2) L}$ correspond to $20 \%$ of the $\mathrm{Cr}$ atoms. Configuration $\mathrm{C}_{1(0.2) L}$ is obtained when the $\mathrm{Cr}$ atoms are placed along a zigzag path of the hexagonal $\mathrm{MoS}_{2}$ monolayer occupying the same Mo sublattice (see figure. 6(a)) and configuration $\mathrm{C}_{2(0.2) L}$ is formed by substituting the Mo atoms along the diagonal of the supercell, also occupying the same Mo sites (see figure. 6(b)).

Configurations $\mathrm{C}_{1(0.4) L}$ and $\mathrm{C}_{2(0.4) L}$ correspond to $40 \%$ of the $\mathrm{Cr}$ atoms. In $\mathrm{C}_{1(0.4) L}$, the $\mathrm{Cr}$ atoms constitute two adjacent parallel lines along the zigzag path (see figure. 6(c)). Configuration $\mathrm{C}_{2(0.4) L}$ is constructed by two lines of the $\mathrm{Cr}$ atoms along the zigzag, but separated by a line of the Mo atoms (see figure. 6(d)). At $40 \%$ concentration, the line-ordered alloy along the diagonal is not possible. Following the same pattern, configurations $\mathrm{C}_{1(0.6) L}$ and $\mathrm{C}_{2(0.6) L}$ are identified in $60 \%$ concentration (see figure. 6(e) and figure. 6(f)). Lastly, configurations $\mathrm{C}_{1(0.8) L}$ and $\mathrm{C}_{2(0.8) L}$ are obtained when $80 \%$ of the $\mathrm{Cr}$ atoms substitute the Mo atoms. These configurations are similar to those of $\mathrm{C}_{1(0.2) L}$ and $\mathrm{C}_{2(0.2) L}$ but the transition metal elements are swapped (as shown in figure. 6(g) and figure. 6(h)). It is clear that at each $\mathrm{Cr}$ concentration, only two distinct line-ordered alloy configurations are possible.

For random alloys, many unique configurations at each con- centration are identified using SQS method. figure. 7 presents the selected unique configurations at $20 \%$ and $40 \%$ concentrations. For a perfect alloy (optimum pair correlation function of the SQS) at $20 \%$ concentration, three $\mathrm{Cr}-\mathrm{Cr}$ bonds $\left(N_{B B}=\right.$ 3) should be formed at each configuration as described in the methodology. For instance in configuration $\mathrm{C}_{1(0.2) R}$ shown in figure. 7(a), the three $\mathrm{Cr}$ atoms adjacent to each other form two $\mathrm{Cr}-\mathrm{Cr}$ bonds $\left(N_{B B}\right)$ and the third $\mathrm{Cr}-\mathrm{Cr}$ bond is formed relatively far away. In configuration $\mathrm{C}_{2(0.2) R}$, three of the introduced $\mathrm{Cr}$ atoms substitute the three Mo atoms in the hexagonal ringlike showing a triangular arrangement, forming the required three bonds (figure. 7(b)). The remaining two $\mathrm{Cr}$ atoms do not necessarily need to form $N_{B B}$ (Cr-Cr bond). Following the same procedure, configurations $\mathrm{C}_{3(0.2) R}, \mathrm{C}_{4(0.2) R}, \mathrm{C}_{5(0.2) R}$, $\mathrm{C}_{6(0.2) R}, \mathrm{C}_{7(0.2) R}$ and $\mathrm{C}_{8(0.2) R}$ are also identified. The same procedure (SQS model for nearest-neighbor pair) has been applied at $40 \%$ concentration. Hence, at $40 \%$, the required $N_{B B}=12$ has been met and all the identified configurations are also presented in figure. 7. At $60 \%$ and $80 \%$ concentrations, all the selected configurations are the same as those obtained at $40 \%$ and $20 \%$, respectively, but with transition metals swapped. Some of our configurations are the same as those in ref. [58] for $\mathrm{Mo}_{(1-x)} \mathrm{W}_{x} \mathrm{~S}_{2}$.

In order to compare the relative stabilities of the $\mathrm{Cr}$ lineordered alloys and random alloys, we have calculated the formation energies using equation. 2 . The calculated formation 

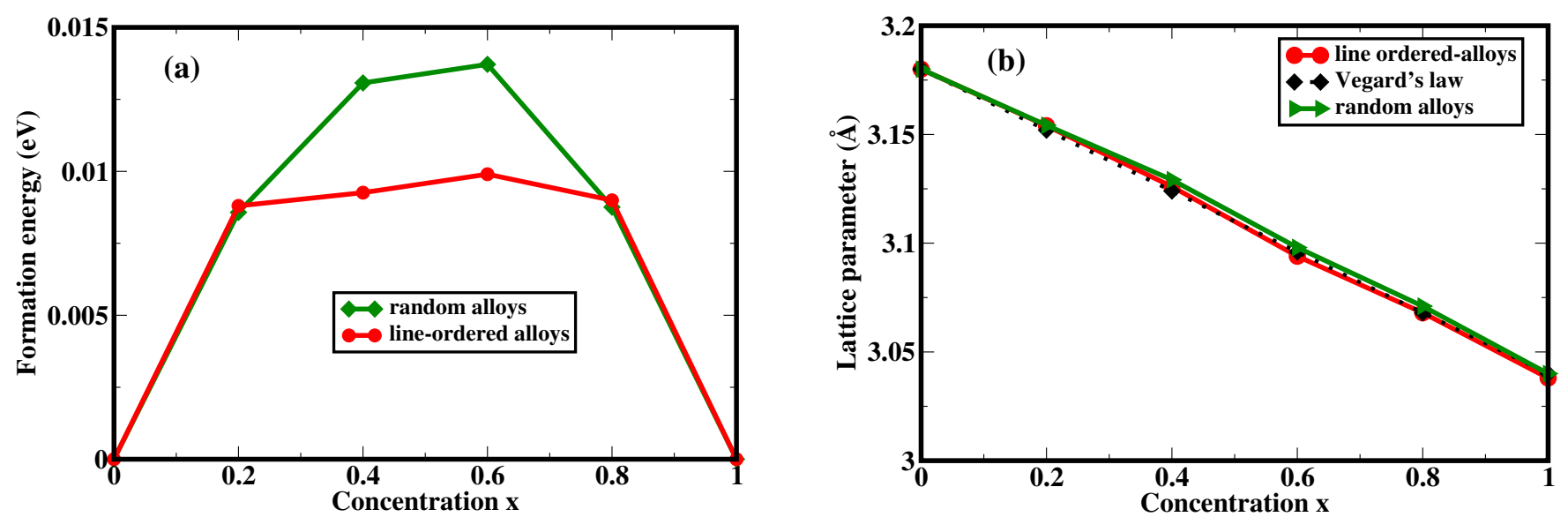

Figure 8: (a) The calculated formation energies of the lowest energy configurations for the Cr line-ordered alloys and random alloys at different concentrations. (b) The lattice constant for $\mathrm{Mo}_{1-x} \mathrm{Cr}_{x} \mathrm{~S}_{2}$ alloys at different concentrations. The red line connects the lowest energy configurations for the line-ordered alloys while the green line connects those for the Cr random alloys.

energies of the line-ordered alloys are presented in the bottom of each diagram in figure. 6 . Although formation of the $\mathrm{Cr}$ lineordered alloys seems to be endothermic (positive formation energy), their magnitude values are relatively very small, suggesting that formation of the $\mathrm{Cr}$ alloys in $\mathrm{a} \mathrm{MoS}_{2}$ monolayer can be spontaneous. Comparing the formation energies of the lineordered alloy configurations at each concentration, we found that $\mathrm{C}_{1(0.2) L}, \mathrm{C}_{1(0.4) L}, \mathrm{C}_{1(0.6) L}$ and $\mathrm{C}_{1(0.8) L}$ are energetically the most stable configurations at $20 \%, 40 \%, 60 \%$ and $80 \%$, respectively. Configuration $\mathrm{C}_{1(0.2) L}$ is $1.1 \mathrm{meV}$ lower than $\mathrm{C}_{2(0.2) L}$ in energy at $20 \%$ concentration. Since the separations between the $\mathrm{Cr}$ atoms are larger in configuration $\mathrm{C}_{2(0.2) L}$ than in configuration $\mathrm{C}_{1(0.2) L}$, this observation suggests that the interaction of $\mathrm{Cr}$ alloys in a $\mathrm{MoS}_{2}$ monolayer is desirable. Moreover, the major driving force on the stability of these systems is the concentration, because figure. 8(a) shows a parabolic curve revealing that the formation energies of the configurations rise with the increase in $\mathrm{Cr}$ or Mo concentration. At $40 \%$, it is noted that configuration $\mathrm{C}_{1(0.4) L}$ is more energetically favorable than configuration $\mathrm{C}_{2(0.4) L}$. This emphasize an idea that the $\mathrm{Cr}$ atoms prefer to be grouped together. It can be seen that when the two $\mathrm{Cr}$ lines alloys are separated by a line of the Mo atoms (see figure. $6(\mathrm{~d})$ ), the relative stability of configuration $\mathrm{C}_{1(0.4) L}$ reduces significantly. This trend is also noted in $60 \%$ of the $\mathrm{Cr}$ atoms. In configuration $\mathrm{C}_{1(0.6) L}$, three $\mathrm{Cr}$ lines alloy are grouped together (see figure. 6(e)) but in configuration $\mathrm{C}_{2(0.6) L}$, three $\mathrm{Cr}$ lines alloy are separated by a line of the Mo atoms (see figure. 6(f)). We observe that configuration $\mathrm{C}_{1(0.6) L}$ is more energetically favorable than configuration $\mathrm{C}_{2(0.6) L}$. At $80 \%$ concentration, we note the same behavior as that of $20 \%$. This is not surprising because in $80 \%$ concentration, the $\mathrm{Cr}$ atoms occupy the sites of Mo in $20 \%$.

Table. 2 presents the stability of the various $\mathrm{Cr}$ random alloy configurations. Just like in the line-ordered alloys, the formation energies of all configurations are positive. Although positive formation energies indicate that $\mathrm{Mo}_{1-x} \mathrm{Cr}_{x} \mathrm{~S}_{2}$ would prefer

Table 2: Formation energies of the selected unique Cr random alloy configurations at different concentrations $x$. The bold values represent the lowest energy configurations at each concentration.

\begin{tabular}{|l|l|l|l|l|}
\cline { 2 - 5 } \multicolumn{1}{c|}{} & \multicolumn{4}{c|}{ Formation energies $(\mathrm{eV})$} \\
\hline Conf. & $x=0.2$ & $x=0.4$ & $x=0.6$ & $x=0.8$ \\
\hline $\mathrm{C}_{1 R}$ & $\mathbf{0 . 0 0 8 6}$ & 0.0139 & 0.0149 & $\mathbf{0 . 0 0 9 0}$ \\
$\mathrm{C}_{2 R}$ & 0.0104 & 0.0181 & 0.0169 & 0.0997 \\
$\mathrm{C}_{3 R}$ & 0.0089 & 0.0149 & 0.0147 & 0.0122 \\
$\mathrm{C}_{4 R}$ & 0.0103 & 0.0144 & 0.0146 & 0.0119 \\
$\mathrm{C}_{5 R}$ & 0.0102 & 0.0185 & 0.0179 & 0.0124 \\
$\mathrm{C}_{6 R}$ & 0.0095 & $\mathbf{0 . 0 1 3 0}$ & $\mathbf{0 . 0 1 4 5}$ & 0.0095 \\
$\mathrm{C}_{7 R}$ & 0.0097 & 0.0182 & 0.0169 & 0.0091 \\
$\mathrm{C}_{8 R}$ & 0.0100 & 0.0167 & 0.0161 & 0.0124 \\
\hline
\end{tabular}


segregation at $0 \mathrm{~K}$ [33], their magnitudes are very small suggesting that they can form at temperatures of few degrees Celsius. These $\mathrm{Cr}$ random alloys are plausible structures and some of these configurations were synthesized at low concentration [57]. The thermodynamic stability of these alloys greatly depends on the $\mathrm{Cr}$ pair coordination $(\mathrm{Cr}-\mathrm{Cr})$, see the lowest energy configuration $\mathrm{C}_{1(0.2) R}$ shown in figure. 7(a). Apart from $\mathrm{C}_{1(0.2) R}$ and $\mathrm{C}_{3(0.2) R}$, all other configurations have slighty high formation energies since they possess an unpaired $\mathrm{Cr}$ atoms. This is in agreement with Wei et al. [33] who reported that the long distance neighbors interaction contribute less energy than nearest-neighbor. Following the pair correlation function, the same behavior is noted at $40 \%$ concentration. The most energetically favorable configuration at $40 \%$ concentration, $\mathrm{C}_{6(0.4) R}$ is shown in figure. 7(n). In this configuration, it is noted that all the $\mathrm{Cr}$ atoms are paired showing $N_{B B}$ correlation. All the selected configurations at $60 \%$ behave as those at $40 \%$. Similar finding is observed also for $20 \%$ and $80 \%$ concentrations. Configurations $\mathrm{C}_{6(0.6) R}$ and $\mathrm{C}_{1(0.8) R}$ to be the most stable at $60 \%$ and $80 \%$ reveal that even Mo alloys in $\mathrm{CrS}_{2}$ monolayer would prefer pair coordination.

The formation energies of the most stable structures at each concentration for the two types of alloys considered are plotted in figure. 8(a). We found that at $x=0.2$ and $x=0.8$, the energy difference between the $\mathrm{Cr}$ line-ordered alloys and $\mathrm{Cr}$ random alloys are very small. This might originates from the fact that in both configurations $\mathrm{C}_{1(0.2) L}$ and $\mathrm{C}_{1(0.2) R}$, the $\mathrm{Cr}$ atoms are completely paired to each other enhancing the stability. However, at $x=0.4$ and $x=0.6$, the line-ordered alloys have significant lower formation energy than the random alloys. The most stable configurations for the $\mathrm{Cr}$ line-ordered alloys $\mathrm{C}_{1(0.4) L}$ and $\mathrm{C}_{1(0.6) L}$ present a continuous $\mathrm{Cr}$ atom pair coordination, whereas in the $\mathrm{Cr}$ random alloy configurations $\left(\mathrm{C}_{6(0.4) R}\right.$ and $\left.\mathrm{C}_{6(0.6) R}\right)$, there is a Mo line separating the group of paired $\mathrm{Cr}$ atoms compromising the stability. Therefore, our results reveal that the line-ordered alloys are plausible structures and can be synthesized at the same conditions as random alloy structures.

We now study the effect of the Cr line-ordered alloys and random alloys on the structural properties of a $\mathrm{MoS}_{2}$ monolayer. After full relaxation, we note that the incorporation of the $\mathrm{Cr}$ atoms into $\mathrm{MoS}_{2}$ monolayer preserves the hexagonal symmetry of the system but the area changes due to the change in lattice constants. Figure. 8(b) shows how lattice constants of $\mathrm{Mo}_{1-x} \mathrm{Cr}_{x} \mathrm{~S}_{2}$ alloys change as a function of $\mathrm{Cr}$ concentration. We find that the values of the lattice constants for the alloys range between those of pristine $\mathrm{MoS}_{2}$ and $\mathrm{CrS}_{2}$ monolayers in both cases. It is also noted in figure. 8(b) that the lattice constants of the configurations are inversely proportional to the $\mathrm{Cr}$ concentration. This reduction is specifically due to the smaller atomic radius of $\mathrm{Cr}$ atom compared to that of Mo atom. The atomic radii of $\mathrm{Cr}$ and $\mathrm{Mo}$ are 166 picometers and 190 picometers, respectively [59].

Usually, the physical properties (e.g. lattice constant and band gap) of an alloy $\mathrm{Mo}_{1-x} \mathrm{Cr}_{x} \mathrm{~S}_{2}$ as a function of the dopants concentration $x$ are described by the following quadratic equation $[25,31,60]$ :

$$
P(x)=(1-x) P\left(M o S_{2}\right)+x P\left(C r S_{2}\right)-b x(1-x),
$$

where $\mathrm{P}\left(\mathrm{MoS}_{2}\right)$ and $\mathrm{P}\left(\mathrm{CrS}_{2}\right)$ are the physical properties of the pristine $\mathrm{MoS}_{2}$ and $\mathrm{CrS}_{2}$ monolayers, respectively and $b$ is called bowing parameter. It characterizes the degree of deviation from linearity trend.

If $b$ in equation. 6 is equal to zero, the so called Vergard's law is obeyed. This law states that there is a linear relationship between the physical properties of a host material and the concentration of alloys $[61,31]$. Then equation. 6 reduces to:

$$
P(x)=x P\left(N X_{2}\right)+(1-x) P\left(M X_{2}\right)
$$

We plotted in figure. 8(b) the lattice constants of the lowest energy configurations for the line-ordered alloys and random alloys as a function of $\mathrm{Cr}$ concentration. For comparison purpose, we have plotted also in figure. 8(b), the lattice constants of the alloys using equation. 7 . We note that the three plots, for the random and line-ordered alloys and equation. 7, are superimposed on top of each other. It can also be seen in figure. 8(b) 

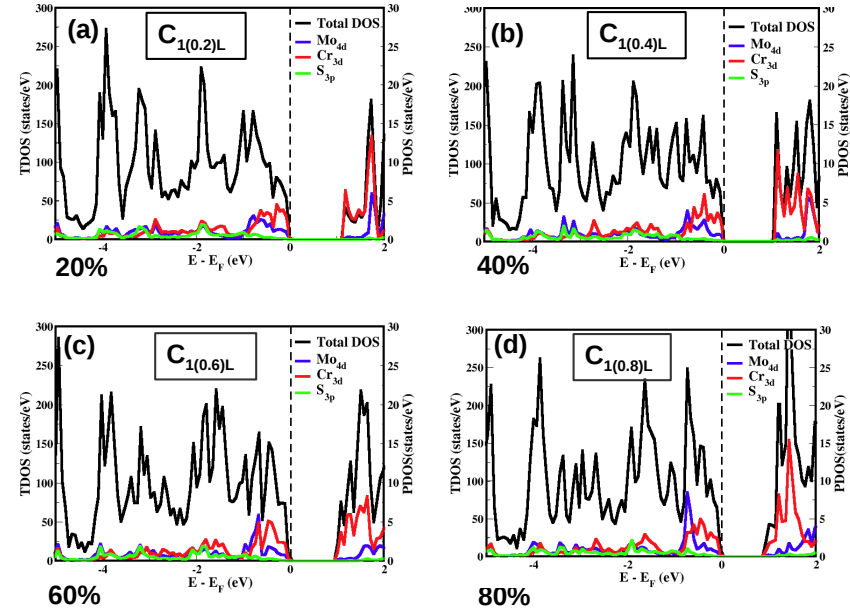

Figure 9: TDOS and PDOS of the stable structures for the Cr line-ordered alloys in a $\mathrm{MoS}_{2}$ monolayer: (a) $\mathrm{C}_{1(0.2) L}$, (b) $\mathrm{C}_{1(0.4) L}$, (c) $\mathrm{C}_{1(0.6) L}$ and (d) $\mathrm{C}_{1(0.8) L}$. The black dashed vertical lines indicate the Fermi level.

that the lattice parameters change almost linearly with the $\mathrm{Cr}$ concentration. This characteristic of the line-ordered and random alloys obeys Vergard's law.

\subsubsection{Electronic properties of $\mathrm{Mo}_{1-x} \mathrm{Cr}_{x} \mathrm{~S}_{2}$ alloys}

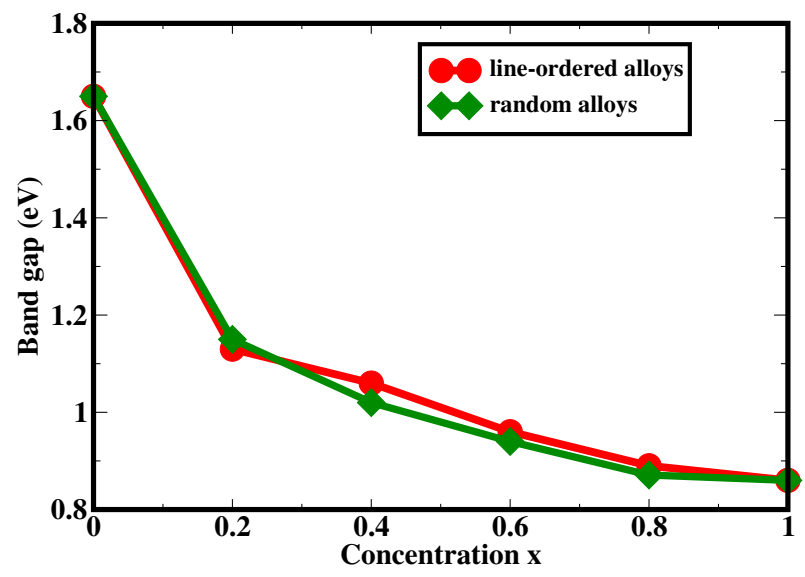

Figure 11: The band gaps of $\mathrm{Mo}_{1-x} \mathrm{Cr}_{x} \mathrm{~S}_{2}$ line-ordered alloys and random alloys as a function of $\mathrm{Cr}$ concentration. Red line connects the band gaps of the most energetically favorable configurations for the line-ordered alloys at each concentration $\mathrm{C}_{1(0.2) L}, \mathrm{C}_{1(0.4) L}, \mathrm{C}_{1(0.6) L}$ and $\mathrm{C}_{1(0.8) L}$, while the green line connects those for the random alloys $\mathrm{C}_{1(0.2) R}, \mathrm{C}_{6(0.4) R}, \mathrm{C}_{6(0.6) R}$ and $\mathrm{C}_{1(0.8) R}$.

To examine the electronic properties of the $\mathrm{Mo}_{1-x} \mathrm{Cr}_{x} \mathrm{~S}_{2}$ alloys, DOS calculations were carried out. Figure. 9 and figure. 10 show the TDOS and PDOS of the stable structures at $20 \%, 40 \%, 60 \%$ and $80 \%$ of the $\mathrm{Cr}$ atoms for the line-ordered
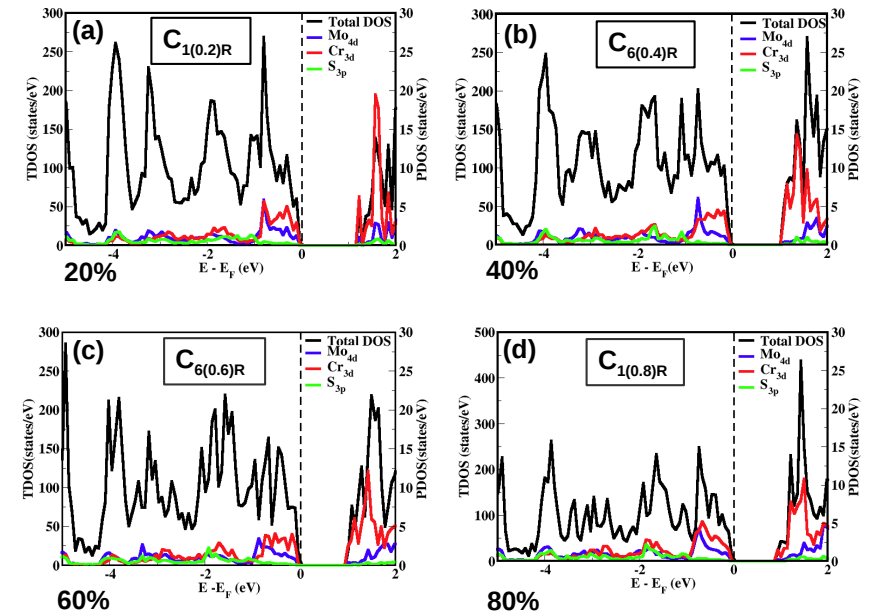

Figure 10: TDOS and PDOS of the stable structures for the Cr random alloys in a $\mathrm{MoS}_{2}$ monolayer: (a) $\mathrm{C}_{1(0.2) R}$, (b) $\mathrm{C}_{6(0.4) R}$, (c) $\mathrm{C}_{6(0.6) R}$ and (d) $\mathrm{C}_{1(0.8) R}$. The black dashed vertical lines indicate the Fermi level.

alloys and random alloys, respectively.

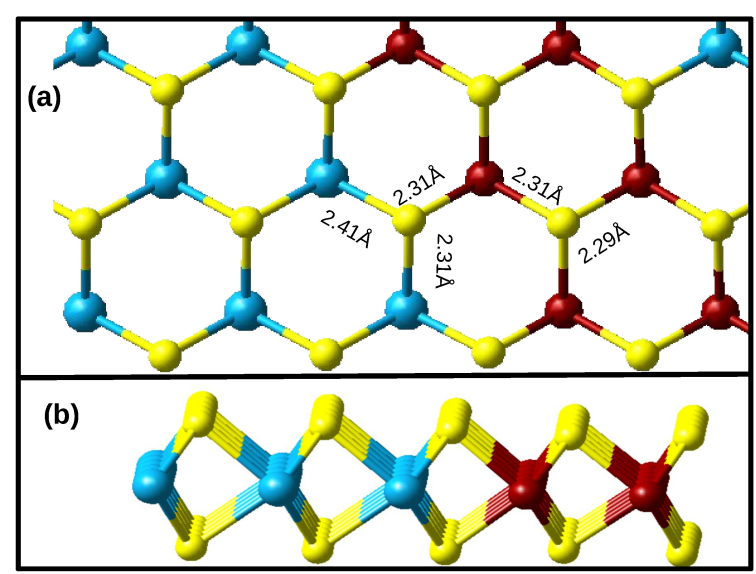

Figure 12: (a)Top view of $\mathrm{Mo}_{1-x} \mathrm{Cr}_{x} \mathrm{~S}_{2}$ line-ordered alloys showing the deviation in bond lengths due to $\mathrm{Cr}$ atoms. (b) Side view of the same $\mathrm{Mo}_{1-x} \mathrm{Cr}_{x} \mathrm{~S}_{2}$ structure. The blue, red and yellow spheres indicate the $\mathrm{Mo}, \mathrm{Cr}$ and $\mathrm{S}$ atoms, respectively.

We find that these two types of alloys significantly fine tune the band gap of a $\mathrm{MoS}_{2}$ monolayer. For all $\mathrm{Cr}$ concentrations considered, the semiconducting behavior of a $\mathrm{MoS}_{2}$ monolayer is preserved but the band gap is reduced. Figure. 11 presents the band gap values of the $\mathrm{Mo}_{1-x} \mathrm{Cr}_{x} \mathrm{~S}_{2}$ alloys calculated at different $\mathrm{Cr}$ concentrations. We find that the band gap decreases when the $\mathrm{Cr}$ concentration increases. The reason might be due 

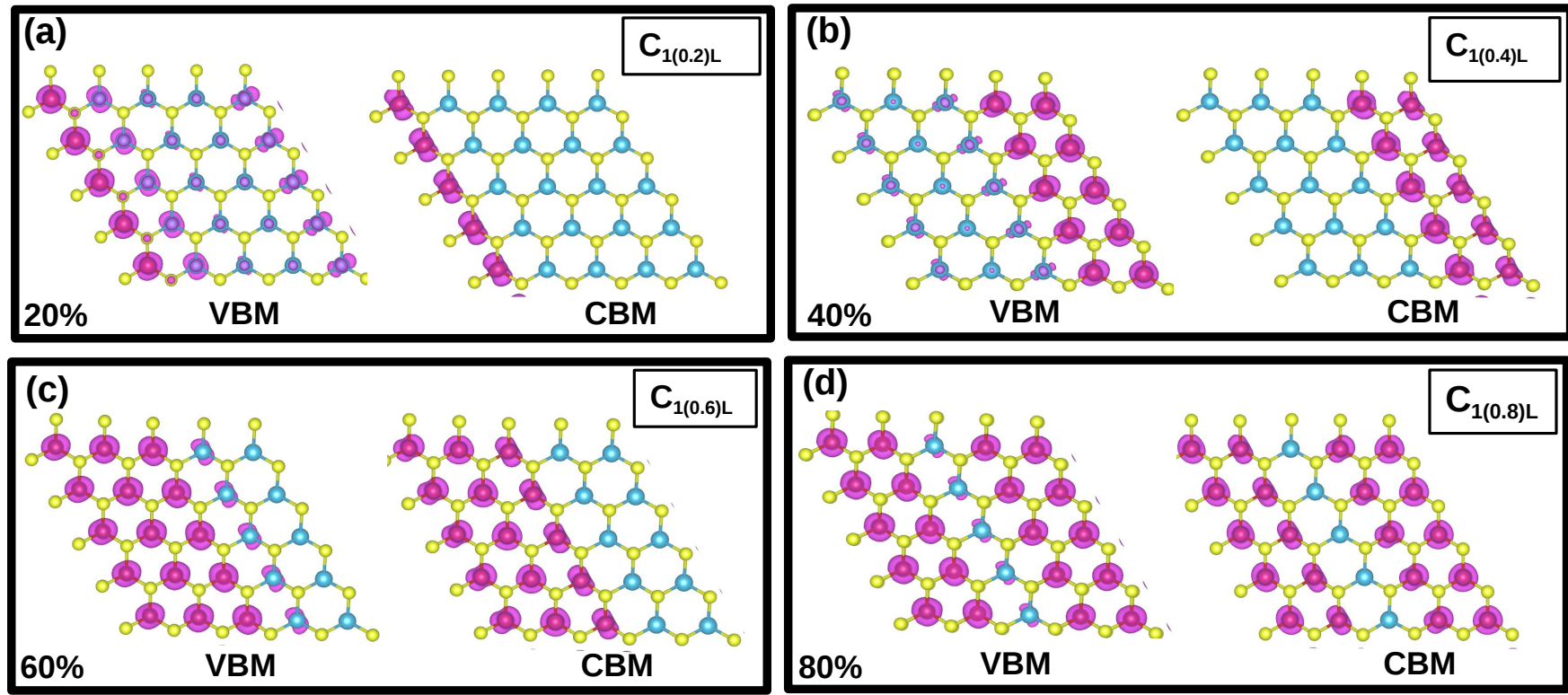

Figure 13: Partial charge densities of the VBM and CBM for the lowest energy line-ordered alloy configurations at (a) 20\%, (b) $40 \%$, (c) $60 \%$ and (d) $80 \%$ Cr concentrations.

to the inward strain along the $x y$-plane induced by small atomic radii of the $\mathrm{Cr}$ atoms compared to those of $\mathrm{Mo}$ atoms. No $\mathrm{Cr}$ buckling is noted along the $z$-axis of the $\mathrm{MoS}_{2}$ monolayer (see figure. 12(b)). Bond lengths of the line-ordered alloys are displayed in figure. 12(a). The relaxed Cr-S bond length is about $2.31 \AA$ on average (same for both line-ordered alloys and random alloys). This value is $0.1 \AA$ less than that of Mo-S of about $2.41 \AA$. The deviation in bond lengths should be responsible for reduction of the $\mathrm{MoS}_{2}$ monolayer band gap as well as the lattice constant. This is opposite to the previous result on isovalent substitution $\mathrm{Mo}_{x} \mathrm{~W}_{1-x} \mathrm{~S}_{2}$, where the band gap increases with the concentration of $\mathrm{W}$ atoms [28]. The band gap of $\mathrm{Mo}_{1-x} \mathrm{Cr}_{x} \mathrm{~S}_{2}$ line-ordered alloys as well as random alloys range between 1.65 $(x=0)$ and $0.86 \mathrm{eV}(x=1)$. This range of band gap is suitable for solar spectrum and thus tuning the band gap of a $\mathrm{MoS}_{2}$ monolayer has an important role in the solar energy conversion [53]. These values might improve when using other exchange-correlation functionals such as HSE or Green's function (GW) [62].

Subsequently, in order to study the contribution of the Cr orbital states on the reduction of band gap, we analyze the PDOS for stable configurations at $20 \%, 40 \%, 60 \%$ and $80 \%$ shown in figure. 9 and figure. 10. This is to examine if there is any hybridization of $\mathrm{Cr}$, Mo and $\mathrm{S}$ orbitals. For all cases, it is noted that the $4 d$ orbitals of the Mo atoms and $3 d$ orbitals of the $\mathrm{Cr}$ atoms are the major contributors for the VBM. The $3 p$ orbitals of the $\mathrm{S}$ atoms also contribute for the VBM but in a small magnitude. We also find that CBM state is mainly contributed by the $3 d$ orbitals of the $\mathrm{Cr}$ atoms. These $\mathrm{Cr} 3 d$ orbitals states get more populated at the band edges when the $\mathrm{Cr}$ concentration increases, thus reducing the band gap significantly as shown in figure. 9(d) and figure. 10(d). We further analyze the partial charge density distribution for VBM and CBM states for the lowest energy line-ordered alloy configurations shown in figure. 13. It is noted indeed that the VBM state is mainly dominated by Mo and $\mathrm{Cr}$ charge densities (see figure. 13(a)). The small cloud of charge on the $\mathrm{S}$ atoms next to $\mathrm{Cr}$ atoms is also noted. On the other hand, the CBM state only arises from the $\mathrm{Cr}$ atoms charge density at each concentration. These partial charge densities results confirm the PDOS analysis.

The GGA exchange correlation is known to severely underestimate the band gap of a real material. In order to check the re- 
liability of our previous GGA calculations, we have calculated the TDOS using HSE functional. For the pristine $\mathrm{MoS}_{2}$ and $\mathrm{CrS}_{2}$ monolayers, our HSE band gaps are $2.17 \mathrm{eV}$ and $1.31 \mathrm{eV}$. Our results are in agreement with the previously reported HSE values (as shown in Table. 1). Compared to the experimental data, HSE overestimates the band gap of $\mathrm{MoS}_{2}$ monolayer $(\sim+0.25 \mathrm{eV})$. Due to the high computational cost consuming of the HSE calculations, we calculated the band gaps of lowest energy configurations. At $20 \%$ of the $\mathrm{Cr}$ atoms, the HSE band gap is $1.62 \mathrm{eV}(1.71 \mathrm{eV})$ for line-ordered (random) alloys more than the GGA value by $0.49 \mathrm{eV}(0.64 \mathrm{eV})$. At $40 \%$, a HSE band gap of $1.53 \mathrm{eV}(1.52 \mathrm{eV})$ has been obtained also more than the GGA predicted value. Although HSE values are more than the GGA values in magnitude, their trends are the same. This suggests that GGA functional can qualitatively describe the line-ordered alloys in a $\mathrm{MoS}_{2}$ monolayer. Our results suggest that $\mathrm{Mo}_{1-x} \mathrm{Cr}_{x} \mathrm{~S}_{2}$ line-ordered alloys are essential for nanotechnology devices.

\section{Conclusions}

In conclusion, using a DFT approach, we have successfully performed a comparative study of the thermodynamic stability, structural and electronic properties of $\mathrm{Mo}_{1-x} \mathrm{Cr}_{x} \mathrm{~S}_{2}$ line-ordered alloys and random alloys. The lowest energy configuration has been identified at each concentration to predict new plausible materials for nanotechnological device applications. The lineordered alloys are constantly lower in formation energies than the random alloys at each concentration. Generally, the formation energies for all configurations are found to be very small (close to zero) suggesting that the $\mathrm{Mo}_{1-x} \mathrm{Cr}_{x} \mathrm{~S}_{2}$ alloys can be synthesized under ambient conditions.

Interestingly, both the line-ordered alloys and random alloys fine tuned the band gap of a $\mathrm{MoS}_{2}$ monolayer. The $\mathrm{Cr}$ concentrations are found to be the major driving force in tuning the band gap of a $\mathrm{MoS}_{2}$ monolayer. The small atomic radii of the $\mathrm{Cr}$ atoms compared to those of Mo atoms induces inward strain in the structure affecting the electronic properties of the $\mathrm{MoS}_{2}$ monolayer. The hybridization of the $\mathrm{Cr} 3 d$ and Mo $4 d$ orbitals occurs at the vicinity of the band edges resulting in the reduction of the band gap. The magnitude of the $\mathrm{Mo}_{1-x} \mathrm{Cr}_{x} \mathrm{~S}_{2}$ alloys band gap values obtained from GGA and HSE functionals meets the requirements for solar energy conversion and nanotechnological devices applications. The further study of lineordered alloys is encouraged for other TMD, both theoretically and experimentally.

\section{Acknowledgment}

The authors acknowledge the University of Pretoria for financial and computational resources support. REM and NC are also grateful to NRF and NITheP for financial support. We also thank R.C. Andrew and O. Olaniyan for useful discussions.

\section{References}

[1] A. K. Geim, K. S. Novoselov, The rise of graphene, Nature materials 6 (3) (2007) 183-191. doi:10.1038/nmat1849.

[2] K. Novoselov, D. Jiang, F. Schedin, T. Booth, V. Khotkevich, S. Morozov, A. Geim, Two-dimensional atomic crystals, Proceedings of the National Academy of Sciences of the United States of America 102 (30) (2005) 10451-10453. doi:10.1073/pnas.0502848102.

[3] H. Zhang, Ultrathin two-dimensional nanomaterials, ACS nano 9 (10) (2015) 9451-9469. doi : 10.1021/acsnano.5b05040.

[4] A. C. Neto, F. Guinea, N. M. Peres, K. S. Novoselov, A. K. Geim, The electronic properties of graphene, Reviews of modern physics 81 (1) (2009) 109. doi:10.1103/revmodphys.81.109.

[5] M. Xu, T. Liang, M. Shi, H. Chen, Graphene-like two-dimensional materials, Chemical reviews 113 (5) (2013) 3766-3798. doi:10.1021/ cr300263a.

[6] K. S. Novoselov, A. K. Geim, S. V. Morozov, D. Jiang, Y. Zhang, S. V. Dubonos, I. V. Grigorieva, A. A. Firsov, Electric field effect in atomically thin carbon films, science 306 (5696) (2004) 666-669. doi:10.1126/ science. 1102896.

[7] O. Olaniyan, R. Mapasha, D. Momodu, M. Madito, A. Kahleed, F. Ugbo, A. Bello, F. Barzegar, K. Oyedotun, N. Manyala, Exploring the stability and electronic structure of beryllium and sulphur co-doped graphene: a first principles study, RSC Advances 6 (91) (2016) 88392-88402. doi : 10.1039/C6RA17640B.

[8] A. Hussain, S. Ullah, M. A. Farhan, Fine tuning of band-gap of graphene by atomic and molecular doping: A density functional theory study, RSC Advances 6 (61) (2016) 55990-56003. doi : 10.1039/c6ra04782c.

[9] P. Rani, V. Jindal, Designing band gap of graphene by B and N dopant atoms, RSC Advances 3 (3) (2013) 802-812. doi:10.1039/ c2ra22664b. 
[10] J. O. Sofo, A. S. Chaudhari, G. D. Barber, Graphane: a two-dimensional hydrocarbon, Physical Review B 75 (15) (2007) 153401. doi:10.1103/ physrevb.75.153401.

[11] X. Gao, Z. Wei, V. Meunier, Y. Sun, S. B. Zhang, Opening a large band gap for graphene by covalent addition, Chemical Physics Letters 555 (2013) 1-6. doi:10.1016/j.cplett. 2012.10.069.

[12] D. Pacile, J. Meyer, C. O. Girit, A. Zettl, The two-dimensional phase of boron nitride: few-atomic-layer sheets and suspended membranes, Applied Physics Letters 92 (13) (2008) 133107. doi : 10. 1063/1. 2903702.

[13] Q. H. Wang, K. Kalantar-Zadeh, A. Kis, J. N. Coleman, M. S. Strano, Electronics and optoelectronics of two-dimensional transition metal dichalcogenides, Nature nanotechnology 7 (11) (2012) 699-712. doi: 10.1038/nnano. 2012.193.

[14] A. Kuc, T. Heine, A. Kis, Electronic properties of transition-metal dichalcogenides, MRS Bulletin 40 (07) (2015) 577-584. doi:10.1557/ mrs.2015.143.

[15] B. Radisavljevic, A. Radenovic, J. Brivio, i. V. Giacometti, A. Kis, Singlelayer $\mathrm{MoS}_{2}$ transistors, Nature nanotechnology 6 (3) (2011) 147-150. doi:10.1038/nnano. 2010.279.

[16] H. Zeng, J. Dai, W. Yao, D. Xiao, X. Cui, Valley polarization in $\mathrm{MoS}_{2}$ monolayers by optical pumping, Nature nanotechnology 7 (8) (2012) 490-493. doi:10.1038/nnano.2012.95.

[17] A. Castellanos-Gomez, M. Poot, G. A. Steele, H. S. van der Zant, N. Agraï, G. Rubio-Bollinger, Elastic properties of freely suspended $\mathrm{MoS}_{2}$ nanosheets, Advanced Materials 24 (6) (2012) 772-775. doi: 10.1002/adma. 201103965.

[18] N. izyumskaya, D. O. Demchenko, V. Avrutin, Ü. ÖZGÜR, H. MorkoÇ, Two-dimensional $\mathrm{MoS}_{2}$ as a new material for electronic devices, Turkish Journal of Physics 38 (3) (2014) 478-496. doi:10.3906/ fiz-1407-16.

[19] H. Wang, H. Yuan, S. S. Hong, Y. Li, Y. Cui, Physical and chemical tuning of two-dimensional transition metal dichalcogenides, Chemical Society Reviews 44 (9) (2015) 2664-2680. doi:10.1039/c4cs00287c.

[20] C. Ataca, H. Sahin, S. Ciraci, Stable, single-layer $\mathrm{MX}_{2}$ transition-metal oxides and dichalcogenides in a honeycomb-like structure, The Journal of Physical Chemistry C 116 (16) (2012) 8983-8999. doi:10.1021/ jp212558p.

[21] M. Berger, Nanotechnology: The Future is Tiny, Royal Society of Chemistry, 2016.

[22] X. Li, H. Zhu, Two-dimensional $\mathrm{MoS}_{2}$ : Properties, preparation, and applications, Journal of Materiomics 1 (1) (2015) 33-44. doi:10.1016/ j.jmat.2015.03.003.

[23] I. Vurgaftman, J. Meyer, L. Ram-Mohan, Band parameters for III-V compound semiconductors and their alloys, Journal of applied physics 89 (11) (2001) 5815-5875. doi : 10.1063/1.1368156.

[24] M. Ameri, A. Bentouaf, M. Doui-Aici, R. Khenata, F. Boufadi, A. Touia, et al., Structural and electronic properties calculations of $\mathrm{Al}_{x} \mathrm{In}_{1-x} \mathrm{P}$ alloy, Materials Sciences and Applications 2 (07) (2011) 729-738. doi:10. 4236/msa.2011.27101.

[25] S. Kumar, S. Joshi, S. Gupta, S. Auluck, Band gap engineering of $\mathrm{CuAl}_{(1-x)} \mathrm{In}_{x} \mathrm{~S}_{2}$ alloys for photovoltaic applications: a first principles study, Journal of Physics D: Applied Physics 49 (20) (2016) 205103. doi:10.1088/0022-3727/49/20/205103.

[26] W. Zhang, X. Li, T. Jiang, J. Song, Y. Lin, L. Zhu, X. Xu, CVD synthesis of $\mathrm{Mo}_{1-x} \mathrm{~W}_{x} \mathrm{~S}_{2}$ and $\mathrm{MoS}_{2(1-x)} \mathrm{Se}_{2 x}$ alloy monolayers aimed at tuning the bandgap of molybdenum disulfide, Nanoscale 7 (32) (2015) 1355413560. doi:10.1039/c5nr02515j.

[27] J. Xi, T. Zhao, D. Wang, Z. Shuai, Tunable electronic properties of twodimensional transition metal dichalcogenide alloys: a first-principles prediction, The journal of physical chemistry letters 5 (2) (2013) 285-291. doi: $10.1021 / j z 402375$ s.

[28] H.-P. Komsa, A. V. Krasheninnikov, Two-dimensional transition metal dichalcogenide alloys: stability and electronic properties, The journal of physical chemistry letters 3 (23) (2012) 3652-3656. doi:10.1021/ jz301673x.

[29] S. Tongay, D. S. Narang, J. Kang, W. Fan, C. Ko, A. V. Luce, K. X. Wang, J. Suh, K. Patel, V. Pathak, et al., Two-dimensional semiconductor alloys: Monolayer $\mathrm{Mo}_{1-x} \mathrm{~W}_{x} \mathrm{Se}_{2}$, Applied Physics Letters 104 (1) (2014) 012101. doi: 10.1063/1.4834358.

[30] L. Xie, Two-dimensional transition metal dichalcogenide alloys: preparation, characterization and applications, Nanoscale 7 (44) (2015) 18392 18401. doi: 10.1039/c5nr05712d.

[31] J. Kang, S. Tongay, J. Li, J. Wu, Monolayer semiconducting transition metal dichalcogenide alloys: Stability and band bowing, Journal of Applied Physics 113 (14) (2013) 143703. doi:10.1063/1.4799126.

[32] L.-Y. Gan, Q. Zhang, Y.-J. Zhao, Y. Cheng, U. Schwingenschlögl, Orderdisorder phase transitions in the two-dimensional semiconducting transition metal dichalcogenide alloys $\mathrm{Mo}_{1-x} \mathrm{~W}_{x} \mathrm{X}_{2}(\mathrm{X}=\mathrm{S}, \mathrm{Se}$, and Te), Scientific reports 4 (2014) 6691. doi:10.1038/srep06691.

[33] X.-L. Wei, H. Zhang, G.-C. Guo, X.-B. Li, W.-M. Lau, L.-M. Liu, Modulating the atomic and electronic structures through alloying and heterostructure of single-layer $\mathrm{MoS}_{2}$, Journal of Materials Chemistry A 2 (7) (2014) 2101-2109. doi : 10.1039/c3ta13659k.

[34] H.-P. Komsa, S. Kurasch, O. Lehtinen, U. Kaiser, A. V. Krasheninnikov, From point to extended defects in two-dimensional $\mathrm{MoS}_{2}$ : evolution of atomic structure under electron irradiation, Physical Review B 88 (3) (2013) 035301. doi:10.1103/physrevb.88.035301.

[35] A. Zunger, S.-H. Wei, L. Ferreira, J. E. Bernard, Special quasirandom structures, Physical Review Letters 65 (3) (1990) 353. doi:10.1103/ physrevlett.65.353.

[36] G. Kresse, J. Hafner, Ab initio molecular dynamics for liquid metals, Physical Review B 47 (1) (1993) 558. doi:10.1103/physrevb.47. 558.

[37] G. Kresse, J. Furthmüller, Efficiency of ab-initio total energy calculations for metals and semiconductors using a plane-wave basis set, Computational Materials Science 6 (1) (1996) 15-50. doi:10.1016/ 
0927-0256 (96) 00008-0.

[38] P. E. Blöchl, Projector augmented-wave method, Physical Review B 50 (24) (1994) 17953. doi:10.1103/physrevb.50.17953.

[39] J. P. Perdew, K. Burke, M. Ernzerhof, Generalized gradient approximation made simple, Physical review letters 77 (18) (1996) 3865. doi : 10.1103/physrevlett.77.3865.

[40] T. L. Tan, M.-F. Ng, G. Eda, Stable monolayer transition metal dichalcogenide ordered alloys with tunable electronic properties, The Journal of Physical Chemistry C 120 (5) (2016) 2501-2508. doi:10.1021/acs . jpcc.5b10739.

[41] A. D. Becke, Density-functional exchange-energy approximation with correct asymptotic behavior, Physical review A 38 (6) (1988) 3098. doi:10.1103/PhysRevA.38.3098.

[42] J. P. Perdew, Density-functional approximation for the correlation energy of the inhomogeneous electron gas, Physical Review B 33 (12) (1986) 8822. doi:10.1103/PhysRevB.33.8822.

[43] C. Lee, W. Yang, R. G. Parr, Development of the colle-salvetti correlationenergy formula into a functional of the electron density, Physical review B 37 (2) (1988) 785. doi : 10.1103/PhysRevB.37.785.

[44] J. P. Perdew, A. Ruzsinszky, G. I. Csonka, O. A. Vydrov, G. E. Scuseria, L. A. Constantin, X. Zhou, K. Burke, Restoring the density-gradient expansion for exchange in solids and surfaces, Physical Review Letters 100 (13) (2008) 136406. doi : 10.1103/PhysRevLett.100.136406.

[45] J. Heyd, G. E. Scuseria, M. Ernzerhof, Hybrid functionals based on a screened coulomb potential, The Journal of Chemical Physics 118 (18) (2003) 8207-8215. doi:10.1063/1.1564060.

[46] C. Jiang, C. Wolverton, J. Sofo, L.-Q. Chen, Z.-K. Liu, First-principles study of binary bec alloys using special quasirandom structures, Physical Review B 69 (21) (2004) 214202. doi:10.1103/physrevb.69. 214202.

[47] X.-L. Fan, Y. Yang, P. Xiao, W.-M. Lau, Site-specific catalytic activity in exfoliated $\mathrm{MoS}_{2}$ single-layer polytypes for hydrogen evolution: basal plane and edges, Journal of Materials Chemistry A 2 (48) (2014) 2054520551. doi:10.1039/c4ta05257a.

[48] D. Yang, S. J. Sandoval, W. Divigalpitiya, J. Irwin, R. Frindt, Structure of single-molecular-layer $\mathrm{MoS}_{2}$, Physical Review B 43 (14) (1991) 12053. doi:10.1103/physrevb.43.12053.

[49] Y. Ding, Y. Wang, J. Ni, L. Shi, S. Shi, W. Tang, First principles study of structural, vibrational and electronic properties of graphene-like $\mathrm{MX}_{2}$ $(\mathrm{M}=\mathrm{Mo}, \mathrm{Nb}, \mathrm{W}, \mathrm{Ta} ; \mathrm{X}=\mathrm{S}, \mathrm{Se}, \mathrm{Te})$ monolayers, Physica B: Condensed Matter 406 (11) (2011) 2254-2260. doi : 10 .1016/j ·physb. 2011.03. 044.

[50] H. Guo, N. Lu, L. Wang, X. Wu, X. C. Zeng, Tuning electronic and magnetic properties of early transition-metal dichalcogenides via tensile strain, The Journal of Physical Chemistry C 118 (13) (2014) 7242-7249. doi:10.1021/jp501734s.

[51] K. F. Mak, C. Lee, J. Hone, J. Shan, T. F. Heinz, Atomically thin $\mathrm{MoS}_{2}$ : a new direct-gap semiconductor, Physical Review Letters 105 (13) (2010)
136805. doi:10.1103/physrevlett.105.136805.

[52] A. Splendiani, L. Sun, Y. Zhang, T. Li, J. Kim, C.-Y. Chim, G. Galli, F. Wang, Emerging photoluminescence in monolayer $\mathrm{MoS}_{2}$, Nano letters 10 (4) (2010) 1271-1275. doi : 10.1021/n1903868w.

[53] M. Faraji, M. Sabzali, S. Yousefzadeh, N. Sarikhani, A. Ziashahabi, M. Zirak, A. Moshfegh, Band engineering and charge separation in the $\mathrm{Mo}_{1-x} \mathrm{~W}_{x} \mathrm{~S}_{2} / \mathrm{TiO}_{2}$ heterostructure by alloying: first principle prediction, RSC Advances 5 (36) (2015) 28460-28466. doi:10.1039/ c5ra00330j.

[54] H. L. Zhuang, M. D. Johannes, M. N. Blonsky, R. G. Hennig, Computational prediction and characterization of single-layer $\mathrm{CrS}_{2}$, Applied Physics Letters 104 (2) (2014) 022116. doi : 10.1063/1.4861659.

[55] K. Dolui, I. Rungger, C. D. Pemmaraju, S. Sanvito, Possible doping strategies for $\mathrm{MoS}_{2}$ monolayers: An ab initio study, Physical Review B 88 (7) (2013) 075420. doi : 10.1103/physrevb.88.075420.

[56] W. S. Yun, J. Lee, Unexpected strong magnetism of cu doped singlelayerMoS $\mathrm{S}_{2}$ and its origin, Physical Chemistry Chemical Physics 16 (19) (2014) 8990-8996. doi:10.1039/c4cp00247d.

[57] D. J. Lewis, A. A. Tedstone, X. L. Zhong, E. A. Lewis, A. Rooney, N. Savjani, J. R. Brent, S. J. Haigh, M. G. Burke, C. A. Muryn, et al., Thin films of molybdenum disulfide doped with chromium by aerosolassisted chemical vapor deposition (AACVD), Chemistry of Materials 27 (4) (2015) 1367-1374. doi : 10.1021/cm504532w.

[58] Y. Chen, J. Xi, D. O. Dumcenco, Z. Liu, K. Suenaga, D. Wang, Z. Shuai, Y.-S. Huang, L. Xie, Tunable band gap photoluminescence from atomically thin transition-metal dichalcogenide alloys, Acs Nano 7 (5) (2013) 4610-4616. doi : 10.1021/nn401420h

[59] E. Clementi, D. Raimondi, W. Reinhardt, Atomic screening constants from scf functions. II. atoms with 37 to 86 electrons, The Journal of chemical physics 47 (4) (1967) 1300-1307. doi : 10.1063/1.1712084.

[60] Y. Huang, X. Chen, D. Zhou, H. Liu, C. Wang, J. Du, L. Ning, S. Wang, Stabilities, electronic and optical properties of $\operatorname{SnSe}_{2(1-x)} \mathrm{S}_{2 x}$ alloys: A first-principles study, The Journal of Physical Chemistry C 120 (10) (2016) 5839-5847. doi:10.1021/acs .jpcc.6b00794.

[61] A. R. Denton, N. W. Ashcroft, Vegards law, Physical review A 43 (6) (1991) 3161. doi:10.1103/physreva.43.3161.

[62] P. Rinke, A. Qteish, J. Neugebauer, C. Freysoldt, M. Scheffler, Combining GW calculations with exact-exchange density-functional theory: an analysis of valence-band photoemission for compound semiconductors, New Journal of Physics 7 (1) (2005) 126. doi:10.1088/1367-2630/ 7/1/126. 